\title{
A SURVEY ON DIFFERENT TYPES OF NOISES AND DE- NOISING TECHNIQUES
}

\author{
Amandeep Kaur ${ }^{1}$, Dr.Vijay Kumar Joshi ${ }^{2}$
}

Abstract: The denoising of image is essential in image processing researches. Image quality could be degraded by unwanted noise during image acquirement or transmission. The main purpose of image denoising is to decrease noise and retain edges of an image. In this paper, various denoising methods are listed which can be implemented to different forms of noises.

Keywords: de-noising techniques, edges, image, image processing.

\section{INTRODUCTION}

The procedure of removing noise from a signal is called denoising.Noise come out with capturing and transfer of image. Noise is unpleasant information that corrupts an image. The traditional way of denoising of an image is filtering and latest is Wavelet. There are two classes of wavelets that are discrete and continuous. Wavelet divide signal into number of signals called as approximation.

\subsection{TYPES OF NOISES}

The noise is characterized by its pattern and by its probabilistic characteristics. There is a wide variety of noise Types while we focus on the most important types, these are Gaussian noise, salt and pepper noise, poison noise, impulse noise, speckle noise.

- Gaussian Noise: Gaussian noise is statistical noise that has its probability density function equal to that of the normal distribution, which is also known as the Gaussian distribution. Principal sources of Gaussian noise in digital images arise during acquisition e.g. sensor noise caused by poor illumination and/or high temperature, and/or transmission e.g. electronic circuit noise.

- Salt and Pepper Noise: Salt and pepper noise is a form of noise typically seen on images. It represents itself as randomly occurring white and black pixels. An effective noise reduction method for this type of noise involves the usage of a median filter, morphological filter or a contra harmonic mean filter.

Salt and pepper noise creeps into images in situations where quick transients, such as faulty switching, take place.

- Poison Noise: Poisson noise is induced by the nonlinear response of the image detectors and recorders. This type of noise is image data dependent. This term arises because Detection and recording processes involve random electron emission having a Poisson distribution with a mean response value. Since the mean and variance of a Poisson distribution Are equal, the image dependent term has a standard deviation if it is assumed that the noise has a unity variance.

- Impulse Noise: Impulse noise is a category of noise which includes unwanted, almost instantaneous sharp sounds. Noises of the kind are usually caused by electromagnetic interference, scratches on the recording disks.

- Speckle Noise: Speckle is a complex phenomenon, which degrades image quality with a backscattered wave appearance which originates from many microscopic diffused reflections that passing through internal organs and makes it more difficult for the observer to discriminate fine detail of the images in diagnostic examinations.

\subsection{DENOISING TECHNIQUES}

There are basic two approaches of the image denoising: spatial domain filtering and transform domain filtering.

1.2.1 Spatial Domain Filtering: Spatial filters are direct and high speed processing tools of images. This is the traditional way to remove the noise from the digital images to employ the spatial filters.

Spatial domain filtering is further classified into linear filters and non-linear filters.

\footnotetext{
${ }^{1}$ Student Masters of Technology, Department of Computer Science and Engineering, LCET, Katani Kalan, Punjab, India

${ }^{2}$ Professor and Head of Department, Department of Computer Science and Engineering, LCET, Katani Kalan, Punjab, India
} 
- Linear filters: Mean filter: Mean filtering is a simple, intuitive and easy to implement method of smoothing images, i.e. reducing the amount of intensity variation between one pixel and the next. It is often used to reduce noise in images. The idea of mean filtering is simply to replace each pixel value in an image with the mean value of its neighbors, including itself. This has the has the effect of eliminating pixel values which are unrepresentative of their surroundings.

- Wiener filter: The wiener filtering method requires the information about the spectra of the noise and the original signal and it works well only if the underlying signal is smooth. Wiener method implements spatial smoothing and its model complexity control correspond to choosing the window size.

\subsubsection{Transform Domain Filter}

- Data-Adaptive Transforms: Recently a new method called Independent Component Analysis (ICA) has gained wide spread attention. One exceptional merit of using ICA is it's assumption of signal to be Non-Gaussian which helps to denoise images with Non-Gaussian as well as Gaussian distribution.

- Non-Adaptive-Transform:

* Wavelet Transform: The wavelet expansion set is not unique. A wavelet system is a set of building blocks to construct or represents a signal or function. It is a two dimensional expansion set, usually a basis, for some class one or higher dimensional signals. The wavelet expansion gives a time frequency localization of the signal. Wavelet systems are generated from single scaling function by scaling and translation. A set of scaling function in terms of integer translates of the basic scaling function by

$\varphi k^{(t)]}=\varphi(t-k) \quad K € z \varphi € L^{2} \ldots \ldots$ (i)

- Fourier transform: Fourier transform theory is of central importance in a vast range of applications in physical science, engineering and applied mathematics. Providing a concise introduction to the theory and practice of Fourier transforms, this book is invaluable to students of physics, electrical and electronic engineering and computer science.

\subsection{Comparison Wavelet Transform with Fourier Transform}

The wavelet transform is often compared with the Fourier transform. Fourier transform is a powerful tool for analyzing the components of a stationary signal (a stationary signal is a signal where there is no change in the properties of signal). For example, the Fourier transform is a powerful tool for processing signals that are composed of some combination of sine and cosine signals (sinusoids) Mallat.

The Fourier transform is less useful in analyzing non-stationary signal (a non-stationary signal is a signal where there is change in the properties of signal). Wavelet transforms allow the components of a non-stationary signal to be analyzed.

The Fourier transform shows up in a remarkable number of areas outside of classic signal processing. Even taking this into account, we think that it is safe to say that the mathematics of wavelets is much larger than that of the Fourier transform. In fact, the mathematics of wavelets encompasses the Fourier transform. The size of wavelet theory is matched by the size of the application area. Initial wavelet applications involved signal processing and filtering. However, wavelets have been applied in many other areas including non-linear regression and compression. An offshoot of wavelet compression allows the amount of determinism in a time series to be estimated.

The main difference is that wavelets are well localized in both time and frequency domain whereas the standard Fourier transform is only localized in frequency domain. The Short-time Fourier transform (STFT) is also time and frequency localized but there are issues with the frequency time resolution and wavelets often give a better signal representation using Multi resolution analysis Walnut.

1.4 Wavelets \& Its TypesThere are many wavelets being used now a day for decomposition of signals and images. The main types/families are daubechies, biorthogonal, coiflets, symlets and dmey. The major characteristics of different types of wavelets/families are briefly described here.

The Daubechies Wavelets are compactly supported and have highest number of vanishing moments. The types are db1, $\mathrm{db} 2 . . . \mathrm{db} 45$. Discrete Wavelet Transform and continuous wavelet transform is possible for these. These are not symmetrical. The length of the filter is $2 \mathrm{~N}$. The number of vanishing moments are $\mathrm{N}$.

The Biorthogonal Wavelets are compactly supported wavelets for which symmetry and exact reconstruction is possible with FIR filters. The types are bior1.1, bior1.3, bior1.5, bior2.2, bior2.4 ...etc. Discrete and continuous wavelet transforms are possible with these.

The Symlets Wavelets are compactly supported wavelets with highest number of vanishing moments. The types are sym1, sym2, sym3, sym4 and sym5. Discrete and continuous wavelet transform is possible with these types of wavelets. Filter length is $2 \mathrm{~N}$.

Coiflets wavelets are compactly supported wavelets with highest number of vanishing moments for both psi and phi for a given support width. Discrete and continuous wavelet transform is possible with these.

Discrete Meyer wavelet is FIR based approx of the Meyer wavelet. Meyer wavelet is infinitely regular orthogonal wavelets. 
2. LITERATURE REVIEW

Rupinderpal Kaur[1] "Survey of De-noising Methods Using Filters and Fast Wavelet Transform" in this paper various noise reduction techniques have been developed for removing noise and retaining edge details in images. Author defined in many de noising techniques. Every technique has its personal assumptions, advantages and limitations. The idea behind these techniques is to acquiesce better results in terms of quality and in removal of different noises. This paper almost covers the de noising techniques.

Øyvind Ryan[2] "Applications of the wavelet transform in image Processing" in this paper Mathematical methods applied in the most recent image formats are presented. First of all, the application of the wavelet transform in JPEG2000 is gone through. JPEG2000 is a standard established by the same group which created the widely used JPEG standard, and it was established to solve some of the shortcomings of JPEG. Also presented are other recently established images formats having wavelet transform as part of the codec. Other components in modern image compression systems are also gone through, together with the mathematical and statistical methods used.

Rajeev Aggarwal [3] "Noise Reduction of Speech Signal using Wavelet Transform with Modified Universal Threshold" In this paper, Discrete-wavelet transforms (DWT) based algorithm is used for speech signal denoising. Here both hard and soft thresholding are used for denoising. Analysis is done on noisy speech signal corrupted by babble noise at $0 \mathrm{~dB}, 5 \mathrm{~dB}, 10 \mathrm{~dB}$ and 15dB SNR levels. Output SNR (Signal to Noise Ratio) and MSE (Mean Square Error) is calculated \& compared using both types of thresholding methods. Soft thresholding method performs better than hard thresholding at all input SNR levels. Hard thresholding shows a maximum of $21.79 \mathrm{~dB}$ improvement whereas soft thresholding shows a maximum of $35.16 \mathrm{~dB}$ improvement in output SNR.

M. Sifuzzaman [4] "Application of Wavelet Transform and its Advantages Compared to Fourier Transform" in this paper Wavelet analysis is an exciting new method for solving difficult problems in mathematics, physics, and engineering, with modern applications as diverse as wave propagation, compression, signal processing, image processing, pattern recognition, computer graphics, the detection of aircraft and submarines and other medical image technology. Wavelet transform of a function is the improved version of Fourier transform. Fourier transform is a powerful tool for analyzing the components of a stationary signal. But it is failed for analyzing the non stationary signal where as wavelet transform allows the components of a non-stationary signal to be analyzed. In this paper, our main goal is to find out the advantages of wavelet transform compared to Fourier transform.

\section{CONCLUSION}

For scientists and engineers noise removal has become a permanent issue. Previous noise removal techniques have been used for many years for their simplicity. Wavelet transform have been used for multiresolution, multiscale quality and application dependent. This approach increases flexibility when applying wavelet transforms and significantly reduces the memory requirements.

\section{REFERENCES}

[1] R. K and R.K “Survey of De-noising Methods Using Filters and Fast Wavelet Transform” International Journal of Advanced Research in Computer Science and Software Engineering, Volume 3, Issue 2, pp-133-136,February 2013.

[2] Ø. Ryan "Applications of the wavelet transform in image Processing" Sponsored by the Norwegian Research Council, project nr. 160130/V30,pp-1-16,nov2004.

[3] R. Aggarwal "Noise Reduction of Speech Signal using Wavelet Transform with Modified Universal Threshold" International Journal of Computer Applications Volume 20- No.5,pp-14-19, April 2011.

[4] M. Sifuzzaman "Application of Wavelet Transform and its Advantages Compared to Fourier Transform" Journal of Physical Sciences, Vol. 13,pp- 121-134, 2009

[5] Shigong Yu "Video Denoising Using Motion Compensated 3-D Wavelet Transform With Integrated Recursive Temporal Filtering" IEEE Transactions on Circuits and Systems for Video Technology, Volume:20,pp. 780 - 791,2010.

[6] S. Udomhunsakul "Ultrasonic speckle denoising using the combination of wavelet transform and Wiener filter", The Second Asian and Pacific Rim Symposium on Biophotonics,pp-195-196,2004.

[7] Moussa Olfa "Ultrasound image denoising using a combination of bilateral filtering and stationary wavelet transform" First International Image Processing, Applications and Systems Conference (IPAS),pp-1 - 5, 2014.

[8] Ajay Boyat "Image denoising using wavelet transform and median filtering" International Conference on Engineering,pp-1-6,2013.

[9] Ajay Kumar Boyat "Image denoising using wavelet transform and wiener filter based on log energy distribution over Poisson-Gaussian noise model" IEEE International Conference on Computational Intelligence and Computing Research,pp-1-6,2014.

[10] V. Seena "A review on feature extraction and denoising of ECG signal using wavelet transform" International Conference on Devices, Circuits and Systems,pp-1-6,2014.

[11] Akash kethwas"Image de-noising using fuzzy and wiener filter in wavelet domain” IEEE International Conference on Electrical, Computer and Communication Technologies" pp-1-5, 2015. 